\title{
REMOTE MONITORING OF PROCESS IN INDUSTRY USING ARM7 AND WIRELESS SENSOR NETWORK
}

\author{
Harshal Ramtekkar ${ }^{*}$, Prof. Divya Meshram² \\ *1,2P.C.E. Nagpur \\ *IEmail: ramtekkarharshal@gmail.com, ${ }^{2}$ Email: divyameshram@gmail.com
}

*Corresponding Author: -

Email: ramtekkarharshal@gmail.com

\begin{abstract}
: -
Industries are setting new standards for processes, which are crucial to meet the challenges. These challenges include gamut of processes for monitoring, precision, safety and many more. In this paper the monitoring of physical parameter at real time environment is proposed. The scheme implemented provides remote monitoring of process in industry using ARM7 controller and Wireless Sensor Network. For long distance monitoring at working place we used ZigBee which will be responsible for transmitting and receiving the sensed values from the parallel processors. With the help of ARM7 controller the data of interest variation in light, gas and temperature is sensed and transmitted to remote mobile phone and PDA's, which makes it remote monitoring application. Practical implementation of the scheme makes its use apparent for monitoring.
\end{abstract}

Index terms: -ARM7, Parallel Processing, ZigBee, Monitoring, Wireless Sensor Network

\section{(c) $\$$ (ㅇ) (1)}




\section{INTRODUCTION}

Monitoring can be defined as the collection, analysis and interpretation of data to effectively grab, manage or alter site management activities. To minimize the environmental impacts associated with activities monitoring proves an important management tool. It is used by industry to collect information to characterize changes in environment to take appropriate action when data indicates that the quantity and/or nature of emissions are changing.

There are different types of monitoring like monitoring of ore before manufacturing the product by checking different parameters; this comes underoffline monitoring i.e., noreal time monitoring. During real time monitoring that is in real time environment, it needs to monitor different physical parameters like temperature, pressure, light, exotic gases, humidity etc for different applications like home security, Industrial automation, Hospitals etc.

Automation in Industries is gaining its significance with the advent of time. The production limits are to be increased and modified to meet the needsin theindustry. But the precision in manufacturing has to be justified every now and then. Thus keeping in view the different processes in industry, the monitoring, controlling of each section involved in the industry is a challenge to be met. It certainly involves many resources. Prominently it involves a large amount of man power and timeconsumption.

Wireless Sensor Networks are still a classical environment monitoring, home automation and the internet of things to industrial automation scenarios.

This paper shows a novel setup for the monitoring of processinindustryusingARM7andWSN, whereZigBeeisthe medium of WSN. Control systems like central controller is connected with several serial devices where central controller communicates with each serial device, process data and interacts between user and real time system. This is best suited only when there are minimum devices and required transmission rate is low. When serial devices are more and higher transmission rate is required at that time processing is complex and performance degrade. In another condition, if the distance between central controller and serial devices is more, so the required length of wiring increases which drop the communication quality. Several parallel processing systems creates complexity of cabling, difficult to identify the actual disconnected node. With the help of sensor nodes and central controller with wireless transmissionandZigBeetechnologyit is possible to minimize the complexity and power consumption of automated control systems.

The rest of the paper is organized as follows. Section II shows the literature review for this paper. Section III gives the brief about the proposed scheme where the central controller and node circuits are shown. The different componentsusedinscheme are described as building blocks in section IV. In section V the Interconnections to build the circuit are discussed. In section VI experiments are performed to evaluate the result. Paper is closed by drawing the conclusion in section VII.

\section{LITERATURE SURVEY}

Li Zheng [3], suggested an IEEE 802.15.4/ZigBee wireless sensor network and reports on its experimental results of a wireless sensor network in industrial automation facilities of Japan. It focuses on the ZigBee wireless sensor network, which can perform well even in heavy industrial environments. When implemented a test in Japan, it is observed that performance and reliabilities for the proposed wireless sensor networks are good enough for some monitoring and non-critical instrument systems.

Liu Yanfei et al [4], presents an improved design of ZigBee Wireless Sensor Network. It highlighted that, the coordinator only deal with the task on theZigBeenetwork, therest tasks will be processed by another processor. The processor is connected with the Coordinator by RS-232 interface. All data information will be sent to the processor though the serial port. So the processor undertakes the task to deal with data, conserve the network information, and communicate with the host computer. At last the scheme tested the improved ZigBee wireless network and verified that improved design of the ZigBee wireless sensor network systemisreasonable, the hardware system performance normal, and the software designing improved the working efficiency. The improved design of the ZigBee wireless sensor network gives a concrete form to distributed processing manner, that improved hardware platform provided good function for the software designing. It comes to know that this designing is very suitable for the large-scale wireless network.

Lei Chen and Shuang Yang [7], aimed at monitoring information of HCHO, CH4, LPG and other toxic and harmful gas concentrations in the chemical production plant. The monitoring system designed composed of a ZigBee wireless sensor network composed of CC2430, MC114 and MS11OO gas sensor, which built a safe, low power consumption, flexible detection system of toxic and harmful gas concentrations. It connected the WSN and internet to implement the data transmission with internet. The implementation design and operation of testing of gas concentration monitoring system can be actualized to save energy, reduce environmental pollution, and promptly and effectively to avoid personnel injuries, so it has practical value, and economic benefits. The system not only applies to toxic and harmful gas monitoring, but also is more suitable for hotels decoration, and it fully embodies advantages of the ZigBee technology in the monitoring field. After the emergence of PC and the Internet, there would be an emergence of a new technology in front of people which would influence the future of human work and lifestyle.

Luo Qinghua et al [10] adopt a wireless parallel test system based on ZigBee. A master device set up a ZigBee wireless network and many slave devices. When the test program starts the test commands are sent to master device in PC via UART port while the master device transmits it to every slave device. After receiving this message, every slave device transmits the test command to the BIT (built in test) which willbetested. AftertheBIT, everyDUT(Device underTest) will return the test result to control PC with the help of master device. The results of the experiments show the system has a low power feature, good communication performance and encryption function. 


\section{PROPOSED SCHEME}

This section describes the details for the design of the monitoring of process in industry using ARM7 and WSN. As shown in figure 1 and figure 2 the system deigns consist of various components, similarly the node design is composed of several sensors of interest. The whole system is divided into two parts- transmitter and receiver. In the transmitter part a network, sensors are used to monitor the physical parameters such as temperature, light, gas. The monitoring data is simultaneously fed to the microcontroller. This data is transmitted efficiently to receiver end through wireless

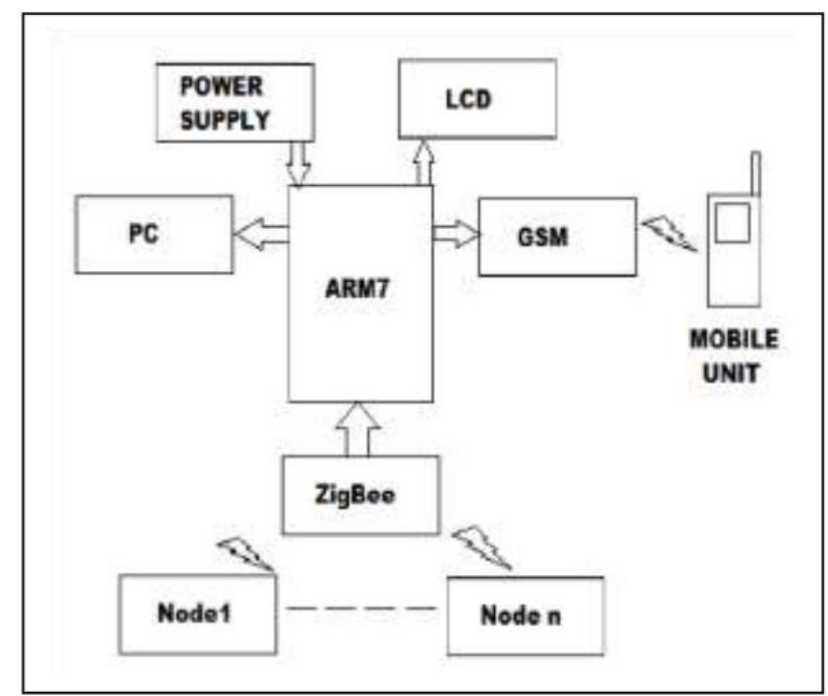

Figure1. System Design

ZigBee Communication Protocol (IEEE802.15.4 Standards). The data received at the receiver end is transferred to ARM7 through MAX232 interface. Thus, a continuous monitoring can be done from a remote location far away from the actual working location.

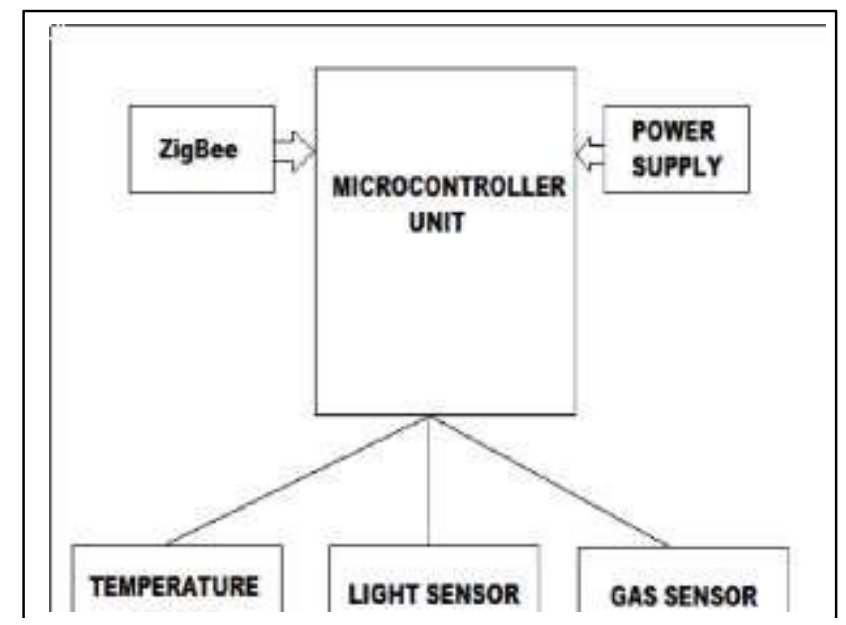

Figure2. Node Design 


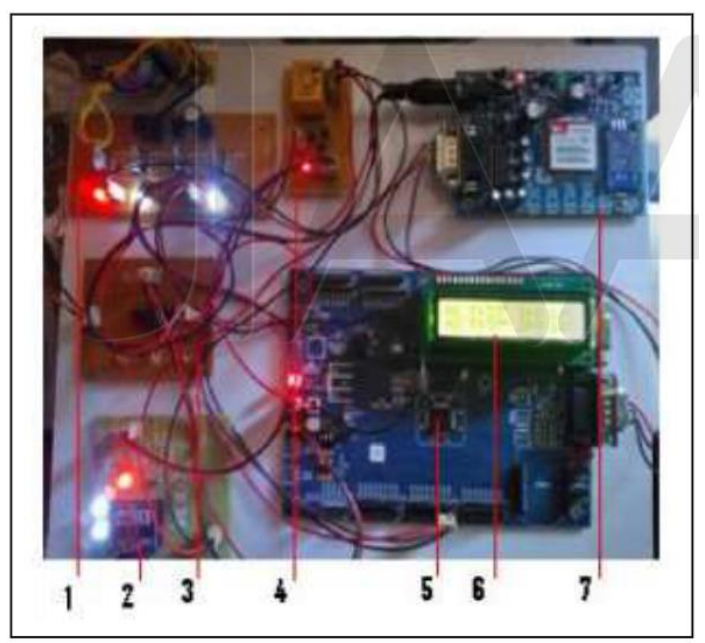

Figure3. Prototype of Central ARM7 Controller

1. Power Supply Circuit.

2. ZigBee Module.

3. MAX232 connection to PC.

4. Relay circui Separating ZigBee and PC connection.

5. LPC2148.

6. $16 \times 2$ LCD Display.

7. SIM900 GSMModule.

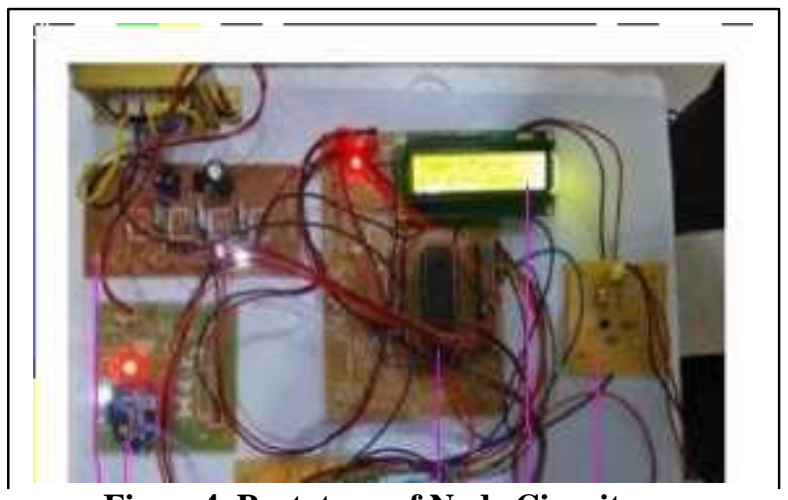

Figure4. Prototype of Node Circuit

1. Power supply circuit

2. ZigBee Module

3. Temperature sensor on ADC Module

4. 0808 ADC Module

5. Microcontroller 89S52

6. Light Sensor Module

7. $16 \times 2$ LCD Display

8. Gas Sensor Module

Thispapercombined the technology ofZigBee,GSMand ARM, where LPC2148 Microcontroller is as the central controller of the automation system. The central controller achieves thefunction of automatic monitoring and timing of the nodes, and take control of real-time dynamic data of individual sensors in monitoring factory application, change in the sensor values will automatically send a message to Central controller and the resolution of command, at the same time GSM module send message to mobile phone at the remote place.

The main aim of this system is to monitor the physical conditions and then send the values to control room by using wireless technology called ZigBee and display them on LCD. The sensors are connected to node controller. Based on sensed values these values are compared with predefined values and corresponding outputs are produced. Every time the sensors monitor different conditions and sensed value is send to Central controller by using ZigBee. ZigBee is interfaced to controller through max232 IC.

\section{BUILDING BLOCKS}

A. ARM Processor

The ARM7TDMI core is a member of the ARM family of general-purpose 32-bit microprocessors. It offers high performance for very low power consumption, and small size. It based on reduced instruction set computerprinciple hence 
much simpler instruction set and related decode mechanism than CISC designs [11].

\section{B. ZigBee}

ZigBee is a wireless communication technology, widely used in wireless sensor network. ZigBee wireless sensor network gives a great advantage in terms of low power consumption, high fault tolerance, flexibility, and autonomy. So it is widely used in military security, environment monitoring, and home automation [4].ZigBeeconsumes very less power between $2 \mathrm{v}$ to $3.6 \mathrm{v}$ and it transfers the data securely. It acts as both transmitter and receiver called as trans-receiver. ZigBee can be either directly interfaced to the microcontroller withoutserial communication cable or with serial communication cable for data serially through wireless communication.

\section{C. $\mu C(A T 89552)$}

The AT89S52 is a low-power, high-performance CMOS 8- bit microcontroller with 8K bytes of in-system programmable Flash memory. The Atmel AT89S52 is a powerful microcontroller provides advantages like high flexibility and cost-effective solution to many embedded control applications. The AT89S52 provides the following standard features: $8 \mathrm{~K}$ bytes of Flash, 256 bytes of RAM, $32 \mathrm{I} / \mathrm{O}$ lines, Watchdog timer, two data pointers, three 16-bit timer/counters, a six-vector two-level interrupt architecture, a full duplex serial port, on-chip oscillator, and clock circuitry. In addition, the AT89S52 is designed with static logicfor operation down to zero frequency and supports two software selectable power saving modes oneisidleandother is power saving mode [5].

\section{D. $L C D$}

In recent years the LCD is finding widespread use replacing LEDs (seven-segment LEDs or other multi segment LEDs). This is due to the following reasons: The prices of LCDs are declining. The ability of display numbers, characters, and graphics. This is in contrast to LEDs, which are limited to numbers and a few characters. Incorporation of a refreshing controller into the LCD relives the CPU of the task for refreshing the LCD. In contrast, the LED must be refreshed by the CPU (or in some other way) to keep displaying the data. Ease of programming for characters and graphics.

\section{E. $A D C 0808$}

The ADC0808, ADC0809 data acquisition component is a monolithic CMOS device. It consists of an 8-bit analog-to- digital converter, 8-channel multiplexer and microprocessor compatible control logic. The converter features a high impedance chopper stabilized comparator, a 256R voltage divider with analog switch tree and a successive approximation register. The 8-channel multiplexer can directly access any of 8 single ended analog signals [12].

\section{F. GSM Sim900}

The SIM900 provides GSM/GPRS 850/900/1800/1900MHz performance for voice, SMS, Data, and Fax in a small form factor and with low power consumption. With atiny configuration of $24 \mathrm{~mm} \times 24 \mathrm{~mm} \times 3 \mathrm{~mm}$, SIM 900 can fit almost all the space requirements in your Mobile-to-Mobileapplication, especially for slim and compact demand of design [13].

\section{G. Sensors Deployed}

Monitoring and controlling physical parameters like temperature, pressure, Gases, light etc. by embedded systems using microcontrollers are very much effective in industrial and research oriented requirements.

\section{1) Gas Sensor}

World coal mine methane (CMM) overview has shown some constraints in India which makes safe mining in country a big challenge. So WSN can be used in mines for safety [6]. In the chemical production process, it would inevitably have the problem of the poisonous gas leak, and ensuring the safe production so it would be particularly suitable for factories to set up wireless sensor networks to detect poisonous gas concentration, making the production safety [7]. The MQ-4 gas sensor can be used to detect gas concentration in the house, and the sensors can be changed into MQ-7 series to detect the concentration of carbon monoxide [1]. MQ-6 gas sensor is used in proposed design. It can detect kinds of flammable gases, especially has high sensitivity to LPG (propane). It is a kind of low-cost sensor for many applications.

\section{2) Temperature Sensor}

Temperature Sensor LM35 is used for sensing temperature. A digital thermometer DS18B20 is another type. Temperature can be monitored through variety of sensor; one should take care in selecting sensors due to different levels of complexity associated with the calibration process. If calibration is notimplemented properly output of the embedded system may vary from actual temperature measured through standard instruments. It detects the temperature changes [8].

\section{3) Light Sensor LDR}

It is very much essential in case of some industrial or home security as well as experimental setup tomonitor as well as controllight continuously. Incase of lightLDR, itserves well but its calibration in Lumens is somehow difficult due to easy unavailability of Lux-meter. Hence in general reference voltage of ADC0808 can be taken with some precautions as intensity of light [8].

\section{INTERCONNECTIONS}

Power supply circuit consist of step-down transformer, bridge rectifier, filter capacitor, and voltage regulator. Step down 
transformer converts $230 \mathrm{v}$ AC into $12 \mathrm{v}$ AC, bridge rectifier converts AC to pulsating $12 \mathrm{v}$ DC, to filter the pulsating DC here capacitor filter is used, and the voltage regulator LM317 regulates $12 \mathrm{v}$ into 3.3v DC which will be the exact voltage supply required for LPC2148 controller. At central controller side this power supply is given to GSM module, Relay, LPC2148, and ZigBee module. At node side power supply is given to ZigBee module, ADC module, $\mu \mathrm{c} 89 \mathrm{~S} 52$, and to Gas sensor module.

LPC2148 board has interconnections with LCD through GPIO, Relay through p0.8 p0.9 p0.10, GSM through UART, PC through external MAX232 with relay switch is ON, ZigBee through relay withrelay is OFF. GSM SIM900module work on ATcommands consist of SIM slot and responsible for sending message to remote mobile phone.

ADC 0808 consist of 8 select analog channels i.e. IN0-IN7 and will be selected using three variables A, B, C. Sensors are connected through three different variable values combination. 555IC sense the sensor value and convert it to sign wave then applied it to ADC circuit, ADC will send this value to $\mu \mathrm{c} 89$ S52. $\mu \mathrm{c} 89 \mathrm{~S} 52$ send it to LCD to display the sensed values and send it to ZigBee through MAX232 IC. MAX232 is connected to the microcontroller(11,12 pin are connected to the 10 and 11 pin i.e. transmit and receive pin of microcontroller).

Atdifferenttimeintervalcentralcontrollerrequesteach node for the sensed values, sensed parameters are acknowledge to the central processor by the node circuit will also use different time interval for different node. It is also important that node circuit is set in such a way that it will sense the parameters at particular intervals.

\section{RESULT}

1. Initially system will prompt to register default mobile number on which it will send the alert message.

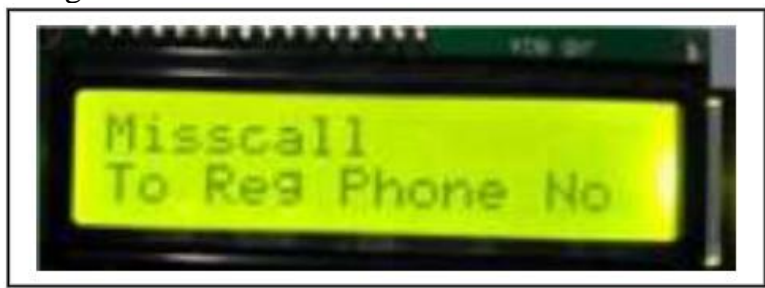

Figure5. Output1

2. After receiving miscall from the remote mobile, it will send the confirmation message.

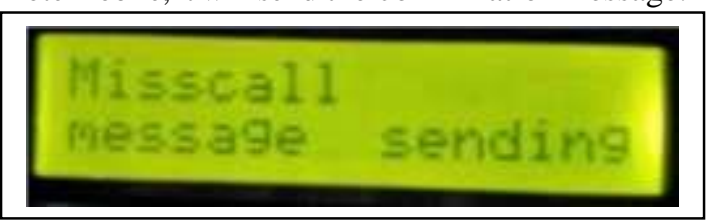

Figure6. Output2

3. Central ARM controller received the sensed values from the two nodes and displaying on LCD.

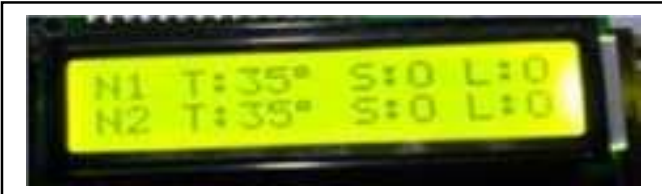

Figure7. Output3

4. Nodecircuit detected low light and smoke, displaying on its LCD screen.

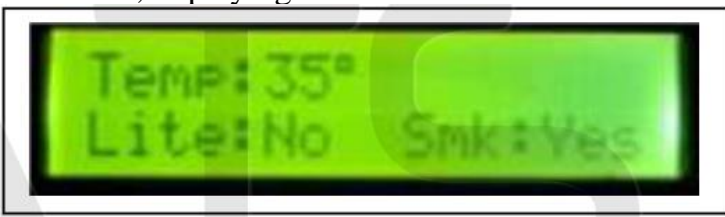

Figure8. Output4

5. Central ARM controller received the sensed value and displayed on itsLCD.

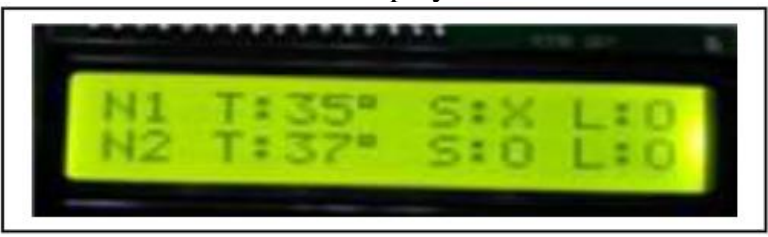

Figure9. Output5 


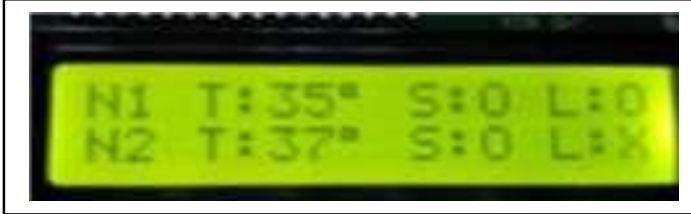

Figure10. Output6

6. At the same time message sent by central controller is received at remote mobile device showing different alerts.

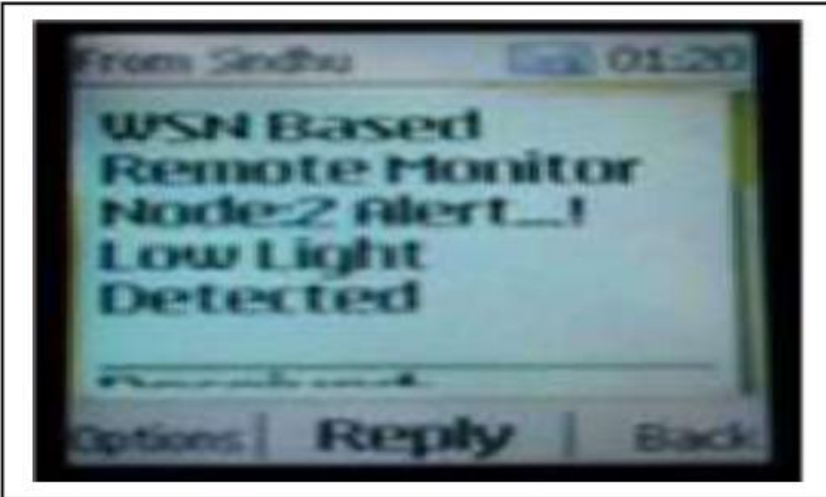

Figure11. Output7

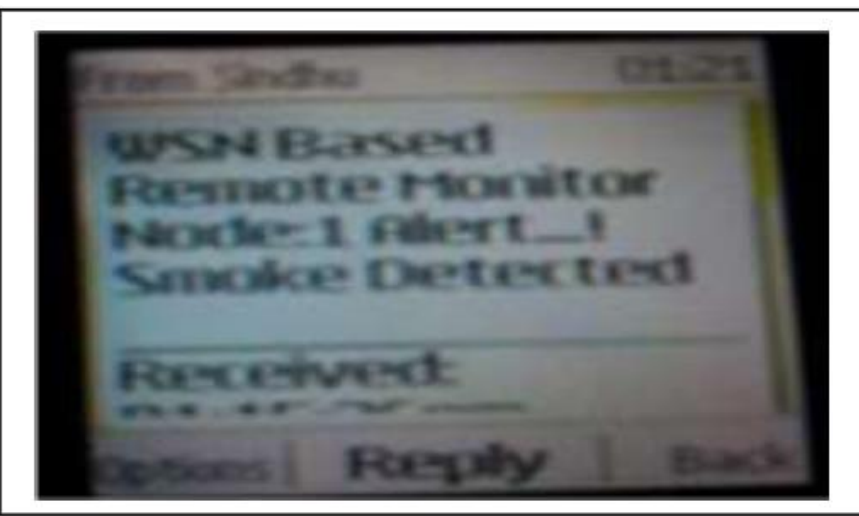

Figure12.Output 8

7. Also the sensed values are displayed on PC using HyperTerminal window.

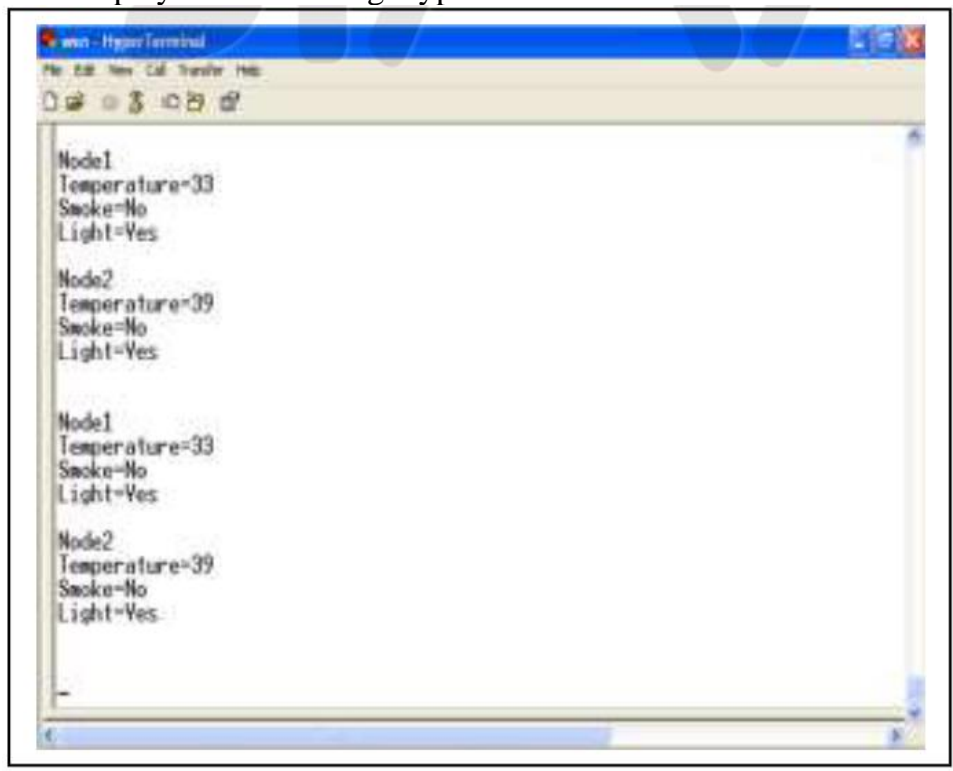

Figure13. Output9 


\section{CONCLUSION}

In this paper, we proposed and successfully implement system design for the monitoring of process in industry. This design consists of a remote monitoring system using ARM7 and ZigBee, in order to monitor processing elements. This novel scheme is designed in two parts namely system design and node design. During the design high performance and low powerfeatures devices were use for sound performance. The result of experiments and evaluation make it apparent that the system is efficient with low power features.

The unique feature of the system is its monitoring through the mobile phones and computer systems. This feature gives an added advantage from implementation point of view and easy remote monitoring and controlling ability. Also result shows, failed sensors are more difficult to detect in a hardware-based closed-loop scheme. Furthermore the system provides the software algorithm logic, by means of which failure can be detectable in efficient and exact manner.

In future we would like to scale the system for various sensor nodes thereby making its use feasible in day to day circumstances such as Body Area Networks, Smart Homes and Devices.

\section{REFERENCES}

[1]. Wu Yi, Wu Tong, Liu Pai ,’ Smart Home System Based on ZigBee and ARM,” ICEMI'2013.

[2]. Dr.Boyina.S.Rao, Deepa.k, Abarna.I, Arthika.S, Hemavathi.G, and Mohanapriya.D, "Controller Area Network for Monitoring and Controlling the environmental parameters using ZigBee communication," International Journal of Advanced Engineering Technology,2012.

[3]. LiZheng,"ZigBee Wireless Sensor Network, "in Industrial Applications Oct. 18-21,2006 in Bexco, Busan, Korea.

[4]. Liu Yanfei Wang ChengQiao Xiaojun Zhang Yunhe, “An Improved Design of ZigBee Wireless Sensor Network,” ICCSIT 2009. 2nd IEEE International Conference.

[5]. Atmel AT89S52 specifications available athttp://www.keil.com/

[6]. Vaibhav Pandit, Prof. U. A. Rane ,” Coal Mine Monitoring Using ARM7 and ZigBee,"in IJETAE ISSN 2250-2459, ISO 9001:2008 Certified Journal, Volume 3, Issue 5, May 2013.

[7]. LeiChen, Shuang Yang,"Based on ZigBee Wireless Sensor Network the Monitoring System Design for Chemical Production Process Toxic and Harmful Gas ," 2010 International conference on CMCE.

[8]. A. Goswami, T. Bezboruah and K.C. Sharma," Design of An Embedded System For Monitoring and Controlling Temperature and Light ,"IJEE November 2009.

[9]. ARM7TDMI, Technical Reference Manual, copyright 1994- 2001.

[10]. Luo Qinghua WeiHai, Weihai, China Yan Xiaozhen;Peng Yu; Peng Xiyuan; Zhang Yujie; Luo Qian,”Design of parallel test system based on ZigBee wireless networks,"Sch. of Inf. \& Electr. Eng., Harbin Inst. of Technol. At IEEE2014.

[11]. ARM7TDMI, Technical Reference Manual, copyright 1994- 2001.

[12]. ADC0808/ADC0809 8-Bit $\mu$ P Compatible A/D Converters with 8-Channel Multiplexer manual by Texas Instruments March 2013.

[13]. Hardware Design SIM900_HD_V1.01 by Shanghai SIM-Com Wireless Solutions Ltd.2009 